\title{
Discussion on Analysis Method of Coplanarity of Marine Steel Structure
}

\author{
Meng Linghe ${ }^{1 *}$, Xiao Liquan ${ }^{1}$, Zhang Shijian ${ }^{1}$, Wang Xuelian ${ }^{2}$ \\ ${ }^{1}$ Surveying Department, Offshore Oil Engineering Co., Ltd., Tianjin, China \\ ${ }^{2}$ Tianjin Ruiyang Offshore Engineering Co., Ltd., Tianjin, China
}

*Corresponding Author: Meng Linghe, Surveying Department, Offshore Oil Engineering Co., Ltd., Tianjin, China

\begin{abstract}
Based on the introduction of traditional flatness error analysis method, this paper introduces a new method using the minimum two-ride fitting technique to propose a new method for the analysis of the coplanarity of marine steel structures. The points are evenly collected on the surface, and the collected points are fitted to an ideal reference plane by least squares method. Finally, the vector distance from each measurement point to the ideal reference plane is calculated, so as to accurately analyze the coplanar surface of the surface to be measured. The coplanarity analysis method introduced in this paper can be extended to the coplanarity analysis of any plane, including the calculation of the coplanarity of the FPSO generator base support plate and the coplanarity analysis of the marine steel structure blind flange.
\end{abstract}

Keywords: coplanarity, optimal plane, measured plane, least squares, mathematical model

\section{INTRODUCTION}

During the construction of offshore oil platforms, turbine generator base support plates and blind plate flanges are widely used, and their coplanarity is related to the normal operation of generators, cranes, pumps and fans, so their coplanarity analysis Very important. Coplanarity is the deviation of the macroscopic concave-convex height of the substrate from the optimal plane, which is a shape error in the row position error. The coplanarity error refers to the variation of the measured actual surface relative to its optimal plane. The actual surface to be measured is compared with the optimal plane, and the line value distance between the two is the coplanarity error.

\section{TRADITIONAL COPLANARITY ANALYSIS METHODS}

\subsection{Maximum Straightness Assessment}

The principle of the maximum straightness evaluation method is to use the maximum straightness error value among all the straight lines in the measured plane as the flatness error [1]. As shown in Figure 1, the measured plane is a square of $100 \mathrm{~m} \times 100 \mathrm{~m}$, and the measurement sampling points are evenly distributed in the square to be measured. The measurement numbers are A, B, C, D, E, F, G, $\mathrm{H}$, I. (See Figure 1a), the relative coordinate value of the $\mathrm{Z}$ coordinate of each measuring point is $\mathrm{mm}$ (see Figure 1b). According to the evaluation principle of the maximum error method, there are six straight lines AC, DF, GI, AG, BH, CI, AI, CG in the plane to be measured, wherein the straight line $\mathrm{CI}$ has the largest straightness error value (see Figure 2), so the plane the flatness error evaluation result is $12 \mathrm{~mm}$.

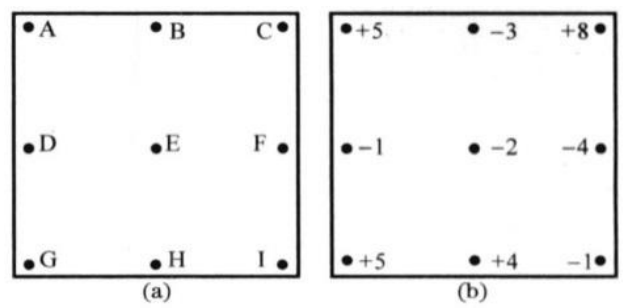

Figure1. Distribution of points to be measured; Data of points to be measured 


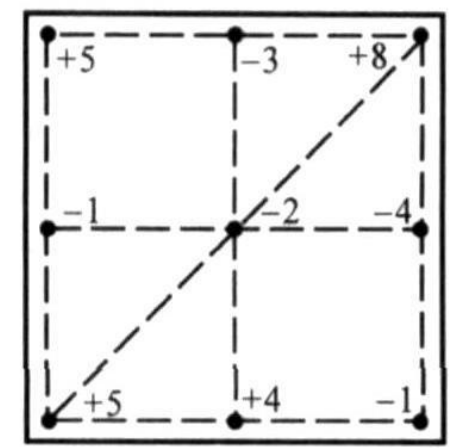

Figure2. Schematic diagram of the maximum straightness evaluation method

\subsection{Diagonal Evaluation}

The diagonal evaluation method is to linearly transform the measured sample point data of the actual measured plane (the $\mathrm{Z}$ coordinate value of each measurement point is simultaneously added or subtracted by a certain value, and the coordinate values of each measurement point are rotated relative to an axis[1-2].The $\mathrm{Z}$ coordinate values on the two diagonals of the transformed plane are equal, and the plane parallel to the two diagonals is used as the optimal plane to evaluate the flatness error of the measured plane. Still taking the measurement data of Figure $1 \mathrm{~b}$ as an example (for comparison purposes, the following various evaluation methods use this set of data), and the transformation process is shown in Figure 3. First, as shown in Figure 3a, as the rotary axis, the $\mathrm{Z}$ coordinate value of point $C$ is subtracted by $115 \mathrm{~mm}$, and the $Z$ coordinate value of point $G$ is added by $115 \mathrm{~mm}$ to obtain the data of Fig. $3 \mathrm{~b}$; and then $0-0$ is shown as Fig. $3 \mathrm{~b}$. For the rotation axis, the $\mathrm{Z}$ coordinate value of point $A$ is subtracted by $3 \mathrm{~mm}$, and the $Z$ coordinate value of point $I$ is added by $3 \mathrm{~mm}$ to obtain the data of Fig. 3c. It can be seen from the data of Fig. 3c that the $\mathrm{Z}$ coordinate values of point $\mathrm{A}$ and point $\mathrm{I}$ on the diagonal $\mathrm{AI}$ of the measured plane are both $+2 \mathrm{~mm}$, and the $\mathrm{Z}$ coordinate values of point $\mathrm{C}$ and point $\mathrm{G}$ on the diagonal $\mathrm{CG}$ are both $+6.5 \mathrm{~mm}$, which is consistent with Diagonal method. The flatness error evaluation result was $11.5 \mathrm{~mm}$.

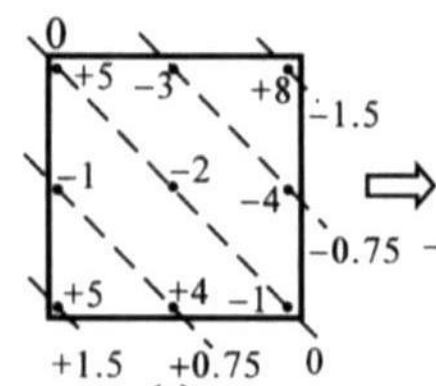

(a)

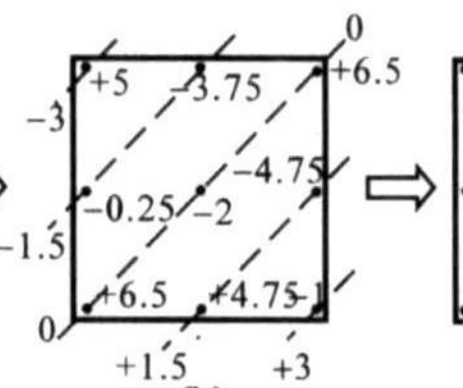

(b)

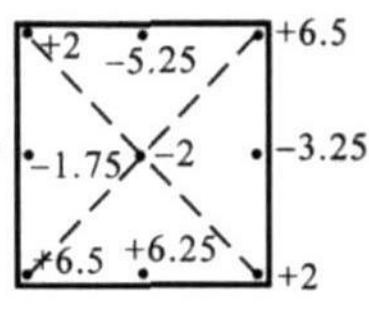

(c)

Figure3. Schematic diagram of the diagonal evaluation method

\subsection{Three-Point Evaluation Method}

The principle of the evaluation method is to linearly transform the measured sample point data of the actual measured plane by a certain form (the $\mathrm{Z}$ coordinate value of each measurement point is simultaneously added or subtracted by a certain value, and the coordinate value of each measurement point is rotated relative to a certain axis), so that The $\mathrm{Z}$ coordinate values of the 3 points in the outermost layer of the transformed plane are equal, and the plane formed by the 3 points is taken as the optimal plane, and the flatness error of the measured plane is evaluated [1-4]. The transformation process is shown in Figure 4. First, take $0-0$ as the rotary axis shown in Fig. 4a, subtract the 115 coordinate value of $\mathrm{C}$ point by $115 \mathrm{~mm}$, and increase the $\mathrm{Z}$ coordinate value of point $\mathrm{G}$ by $115 \mathrm{~mm}$ to obtain the data of Fig. $4 \mathrm{~b}$; then, with 0 - as shown in Fig. $4 \mathrm{~b}$ As the rotary axis, the $\mathrm{Z}$ coordinate of point $\mathrm{A}$ is added to $115 \mathrm{~mm}$, and the $\mathrm{Z}$ coordinate value of point $\mathrm{I}$ is subtracted by $115 \mathrm{~mm}$ to obtain the data of Fig. 4c. It can be seen from the data of Fig. $4 \mathrm{c}$ that the $\mathrm{Z}$ coordinate values of the 3 points $\mathrm{A}, \mathrm{C}$, and $\mathrm{G}$ points of the outermost layer of the measured plane are $+615 \mathrm{~mm}$, which conforms to the three-point evaluation principle, and the flatness error evaluation result is $12 \mathrm{~mm}$. The result is not unique. 


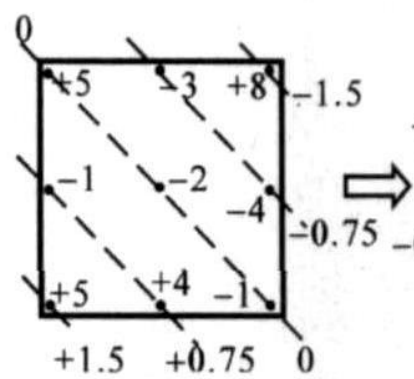

(a)

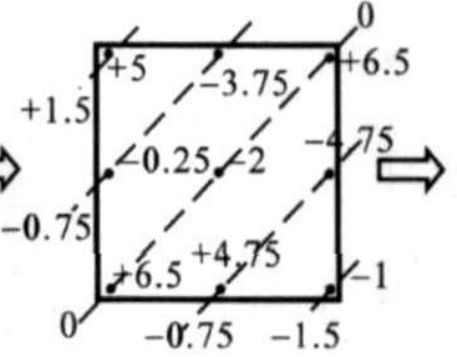

(b)

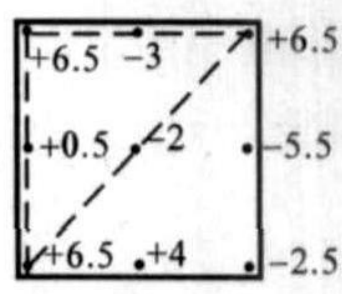

(c)

Figure4. Three-point calibration diagram

\subsection{Minimum Area Assessment}

The principle of the minimum area assessment method is to ensure that two planes parallel to the optimal plane contain the actual plane sampling point data and the distance is the smallest. For the case where the measured point of the plane to be measured is 9 points, the linearly transformed data should satisfy one of the cross criterion, the triangular criterion or the linear criterion [3]. The evaluation result of the obtained flatness error should satisfy the minimum regional condition. The transformation process is shown in Figure 5. First, take 0-0 as the rotary axis shown in Fig. 5a, subtract the 115 coordinate value of $\mathrm{C}$ point by $115 \mathrm{~mm}$, and increase the $\mathrm{Z}$ coordinate value of point $\mathrm{G}$ by $115 \mathrm{~mm}$ to obtain the data of Fig. $5 \mathrm{~b}$; then, with 0 - as shown in Fig. $5 \mathrm{~b} 0$ is used as the rotary axis, and the $\mathrm{Z}$ coordinate value of point $\mathrm{A}$ is subtracted by $1 \mathrm{~mm}$, and the $\mathrm{Z}$ coordinate value of point $\mathrm{I}$ is added by $1 \mathrm{~mm}$ to obtain the data of Fig. 5c. It can be seen from the data of Fig. $5 \mathrm{c}$ that the $\mathrm{Z}$ coordinate data of the two intersecting straight lines $\mathrm{CG}$ and $\mathrm{BF}$ on the measured plane are respectively the maximum value $+615 \mathrm{~mm}$ and the minimum value $-4125 \mathrm{~mm}$, which meets the cross criterion of the minimum area evaluation method, and the flatness error evaluation result is $10.75 \mathrm{~mm}$

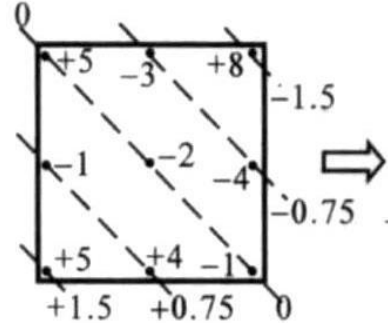

(a)

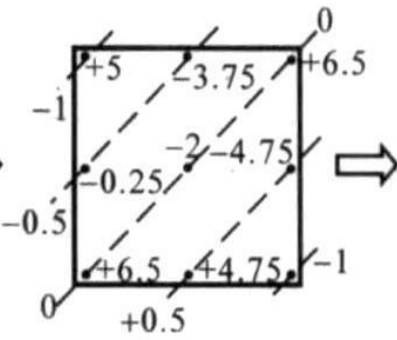

(b)

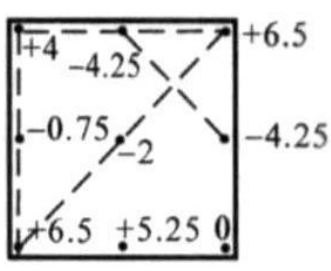

(c)

Figure5. Schematic diagram of the minimum area assessment method

\subsection{Characteristics of Traditional Coplanar Analysis Methods}

The results of the above-mentioned coplanarity evaluation of the same measured plane by various conventional evaluation methods are listed in Table 1 below. It can be seen from the analysis results in the table that the coplanarity error obtained by the minimum area method is the smallest; the coplanarity result obtained by the diagonal evaluation method is about 1.13 times of the coplanarity result obtained by the minimum area method, and the obtained value is the most Close; the maximum value is the same as the value obtained by the three-point evaluation method, which is about 1.18 times of the result obtained by the minimum area method, and the obtained value error value is slightly larger.

Table1. Coplanarity error values obtained by various traditional evaluation methods

\begin{tabular}{|c|c|c|c|c|}
\hline Analytical method & $\begin{array}{c}\text { Minimum area } \\
\text { method }\end{array}$ & Diagonal method & $\begin{array}{c}\text { Three-point } \\
\text { method }\end{array}$ & $\begin{array}{c}\text { Maximum } \\
\text { straightness method }\end{array}$ \\
\hline Coplanar error value & 10.175 & 11.5 & 12 & 12 \\
\hline
\end{tabular}

Comparing the difficulty of data processing of various traditional evaluation methods, the traditional coplanarity evaluation method is characterized by: if the number of data sampling points of the measured plane is small and evenly distributed, the maximum straightness method, the three-point 
method and the pair The data processing of the angular method is quite simple; the results obtained by the three-point evaluation method are not unique, and the data processing of the minimum area method is more complicated, because not all measurement data can satisfy the cross criterion, the triangle criterion or the linear transformation. One of the straight line criteria. Moreover, when the data sampling points of the measured plane are not evenly distributed and the number of data collection points is large, it is quite cumbersome to evaluate the coplanarity error by the maximum straightness method, the diagonal method and the diagonal method, and it is even impossible to evaluate [4-5].

\section{NEW METHOD OF COPLANARITY ANALYSIS}

This paper introduces a method of fitting the optimal plane by least squares method, and calculates the vector distance from the measurement point to the optimal plane on each support plate to determine whether the coplanarity meets the relevant technical requirements and makes adjustments.

\subsection{Establishing a Mathematical Model}

First, we use the total station to collect the coordinates of the point to be measured on the actual surface to be tested in the same coordinate system $\left\{x_{i}, y_{i}, z_{i}\right\}$.

Let us set the equation for the optimal plane as $z=a x+b y+c$.

According to the least squares method, the plane determined when $m=\sum_{i=1}^{n}(z-a x-b y-c)^{2}$ takes the minimum value is the optimal plane we require [6]. In order to find the normal vector $(a, b,-1)$ and $c$ value of the optimal plane, let $\mathrm{m}$ be $\mathrm{a}, \mathrm{b}, \mathrm{c}$ to find the partial derivative equal to zero.

Expand, $m=\sum_{i=1}^{n}\left(z^{2}+a^{2} x^{2}+b^{2} y^{2}+c^{2}-2 a x z-2 b y z-2 c z+2 a b x y+2 a c x+2 b c y\right)$

Then, $\frac{\partial m}{\partial a}=2 a \sum_{i=1}^{n} x_{i}^{2}-2 \sum_{i=1}^{n} x_{i} z_{i}+2 b \sum_{i=1}^{n} x_{i} y_{i}+2 c \sum_{i=1}^{n} x_{i}=0$

$\frac{\partial m}{\partial b}=2 b \sum_{i=1}^{n} y_{i}^{2}-2 \sum_{i=1}^{n} y_{i} z_{i}+2 a \sum_{i=1}^{n} x_{i} y_{i}+2 c \sum_{i=1}^{n} y_{i}=0$

$\frac{\partial m}{\partial c}=2 n c-2 \sum_{i=1}^{n} z_{i}+2 a \sum_{i=1}^{n} x_{i}+2 b \sum_{i=1}^{n} y_{i}=0$

Finished up, $a \sum_{i=1}^{n} x_{i}^{2}+b \sum_{i=1}^{n} x_{i} y_{i}+c \sum_{i=1}^{n} x_{i}=\sum_{i=1}^{n} x_{i} z_{i}$

$a \sum_{i=1}^{n} x_{i} y_{i}+b \sum_{i=1}^{n} y_{i}^{2}+c \sum_{i=1}^{n} y_{i}=\sum_{i=1}^{n} y_{i} z_{i}$

$a \sum_{i=1}^{n} x_{i}+b \sum_{i=1}^{n} y_{i}+n c=\sum_{i=1}^{n} z_{i}$

That is $\left[\begin{array}{ccc}\sum_{i=1}^{n} x_{i}^{2} & \sum_{i=1}^{n} x_{i} y_{i} & \sum_{i=1}^{n} x_{i} \\ \sum_{i=1}^{n} x_{i} y_{i} & \sum_{i=1}^{n} y_{i}^{2} & \sum_{i=1}^{n} y_{i} \\ \sum_{i=1}^{n} x_{i} & \sum_{i=1}^{n} y_{i} & n\end{array}\right]\left[\begin{array}{l}a \\ b \\ c\end{array}\right]=\left[\begin{array}{c}\sum_{i=1}^{n} x_{i} z_{i} \\ \sum_{i=1}^{n} y_{i} z_{i} \\ \sum_{i=1}^{n} z_{i}\end{array}\right]$

Then the matrix of a, b, c values is expressed as,

$\left[\begin{array}{l}a \\ b \\ c\end{array}\right]=\left[\begin{array}{ccc}\sum_{i=1}^{n} x_{i}^{2} & \sum_{i=1}^{n} x_{i} y_{i} & \sum_{i=1}^{n} x_{i} \\ \sum_{i=1}^{n} x_{i} y_{i} & \sum_{i=1}^{n} y_{i}^{2} & \sum_{i=1}^{n} y_{i} \\ \sum_{i=1}^{n} x_{i} & \sum_{i=1}^{n} y_{i} & n\end{array}\right]^{-1} \times\left[\begin{array}{c}\sum_{i=1}^{n} x_{i} z_{i} \\ \sum_{i=1}^{n} y_{i} z_{i} \\ \sum_{i=1}^{n} z_{i}\end{array}\right]$ 
In this way, we get the equation of the optimal plane. After the plane equation is obtained, we can find the vector distance from each measurement point to the optimal plane. The vector distance equation from the measurement point to the optimal plane is:

$$
H_{i}=\frac{a x_{i}+b y_{i}-z_{i}+c}{\sqrt{a^{2}+b^{2}+1}}[9]
$$

According to the value $H_{i}$ of each measurement point, it is possible to judge the extent to which it deviates from the optimal plane. At this point, the parameters of the coordinate transformation are calculated. Then the coplanarity of the plane sought is: $\varepsilon=H_{\max }-H_{\min }$ [8].

\subsection{Programming and Data Testing}

According to the above mathematical calculation principle, the calculation program is written by using MATLAB. We use MATLAB to generate 20 test points evenly and substitute them into the model for calculation. The test data is shown in Table 2 below:

Table2. MATLAB Test Data Sheet

\begin{tabular}{|c|c|c|c|c|}
\hline \multirow{2}{*}{ Point ID } & \multicolumn{3}{|c|}{ Test point coordinates } & Test point deviation \\
\cline { 2 - 5 } & $\mathrm{X}$ & $\mathrm{Y}$ & $\mathrm{Z}$ & $\mathrm{H}$ \\
\hline 1 & 17982 & 1174 & -355.401 & -0.6940307 \\
\hline 2 & 21448 & 1126 & -356.146 & -1.2570577 \\
\hline 3 & 9108 & 1158 & -353.943 & -0.06873258 \\
\hline 4 & 13026 & 1136 & -354.497 & -0.69920359 \\
\hline 5 & 12791 & 8224 & -288.763 & -1.78922219 \\
\hline 6 & 5253 & 1160 & -355.348 & 2.322540589 \\
\hline 7 & 9426 & 8240 & -288.542 & -1.01888392 \\
\hline 8 & 17891 & 8246 & -290.605 & -1.0273335 \\
\hline 9 & 21509 & 8172 & -294.449 & 1.233158451 \\
\hline 10 & 5644 & 8222 & -289.097 & 0.322617671 \\
\hline 11 & 4102 & 7640 & -293.070 & -0.62021691 \\
\hline 12 & 4150 & 4368 & -320.962 & -2.55532304 \\
\hline 13 & 4159 & 1460 & -353.315 & 3.298478027 \\
\hline 14 & 23020 & 7878 & -302.036 & 5.76141583 \\
\hline 15 & 22969 & 4378 & -324.610 & -3.54299014 \\
\hline 16 & 22945 & 1400 & -355.618 & 0.334781499 \\
\hline
\end{tabular}

Import the data points into MATLAB, write the corresponding algorithm, and realize the fitting image of the corresponding data optimal plane in MATLAB as shown in Figure 6:

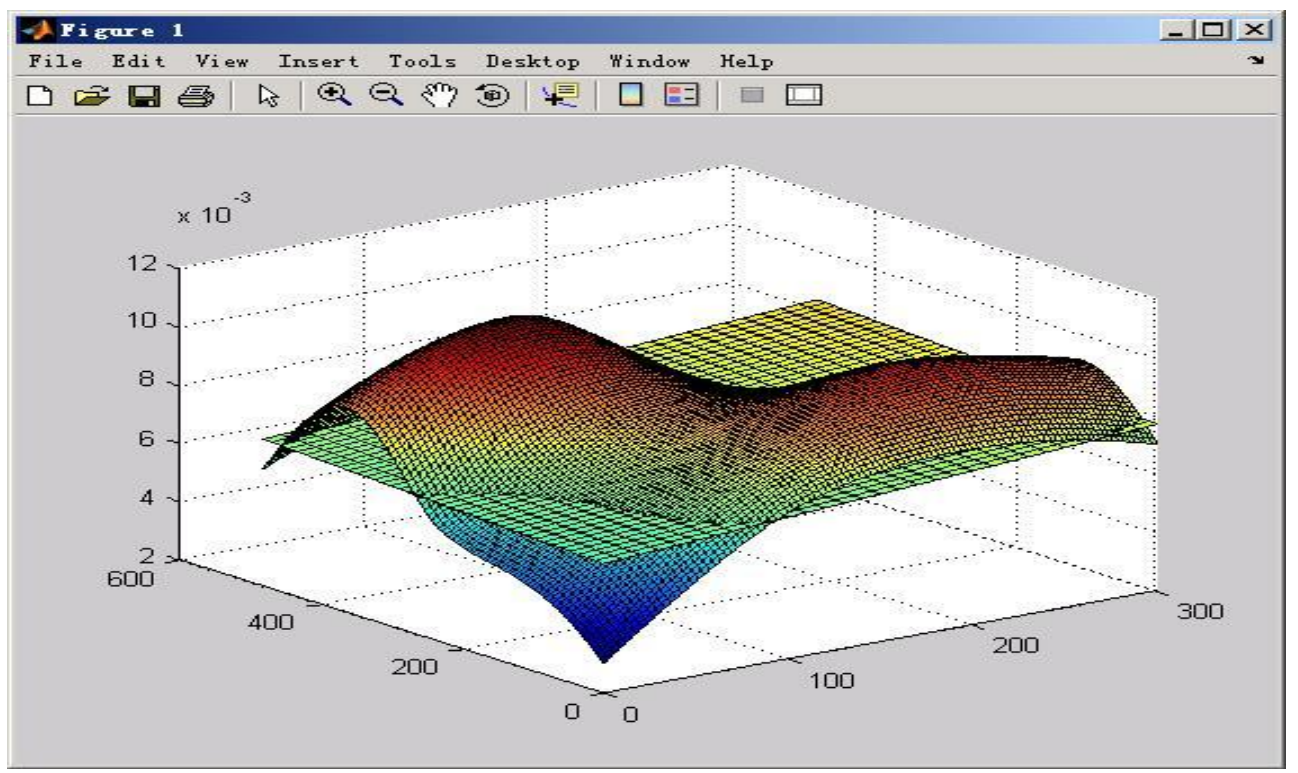

Figure6. MATLAB coordinate fitting program interface 


\subsection{Practical Applications}

The following is the practical application of the algorithm in the offshore oil 112 FPSO turbine generator installation project. Under the premise that the measuring equipment can be stably installed on the hull (that is, the impact of the ship's own vibration on the measuring equipment is within the controllable range), the turbine installation and leveling scheme of the turbine unit can be divided into three stages [7].

\subsubsection{Coplanarity Measurement of Octagonal Plates}

Before the pair of load-bearing plates, it is necessary to measure the coplanarity of the deck part that is docked with it, that is, measure the octagonal plate (as shown below) to ensure that the load-bearing plate can be installed smoothly in the subsequent work. The octagonal board diagram is shown in Figure 7 below:

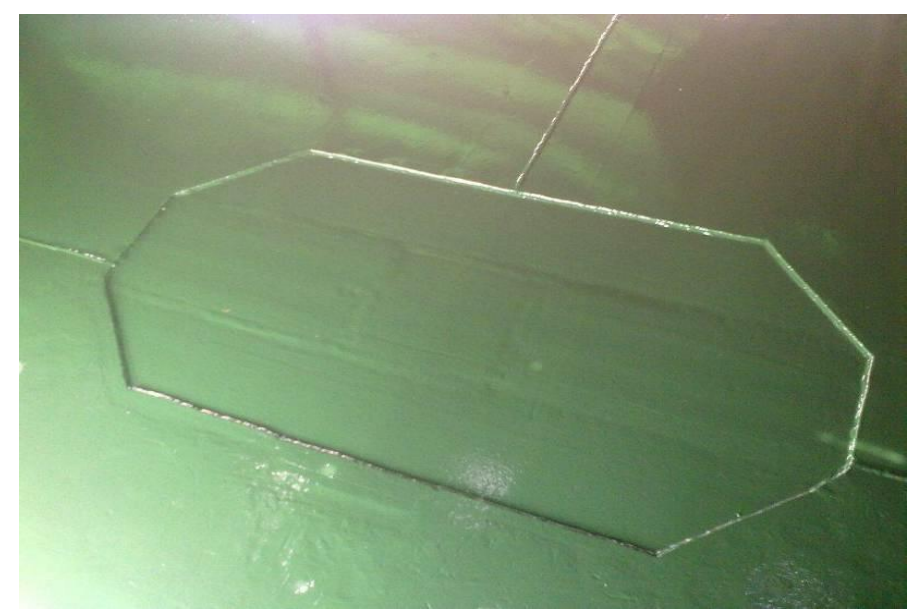

Figure7. Octagonal board

Each octagonal plate is docked with the load-bearing plate at a rectangular area of $1060 \mathrm{~mm} \times 760$ $\mathrm{mm} .9$ points are measured on this area on each octagonal plate, and the coplanarity is characterized by calculating the distance from each point to the optimal plane.

\subsubsection{Bearing Plate Coplanarity Measurement}

After the bearing plate set is paired and after welding, the coplanarity of the upper surface is measured to ensure that the bearing plate can meet the corresponding technical requirements after the welding is completed.

\subsubsection{Coplanarity Measurement of SOLE PLATE}

After SOLE PATE welding, it is necessary to measure the coplanarity of the upper surface to confirm that the corresponding technical requirements have been met after the SOLE PLATE welding is completed. The turbine generator base support plate has three SOLE PLATEs. To ensure a good coplanarity, it is necessary to measure the coplanarity of each SOLE PLATE [11-13]. Find 12 bisectors on each SOLE PLATE, as shown in Figure 8 below:

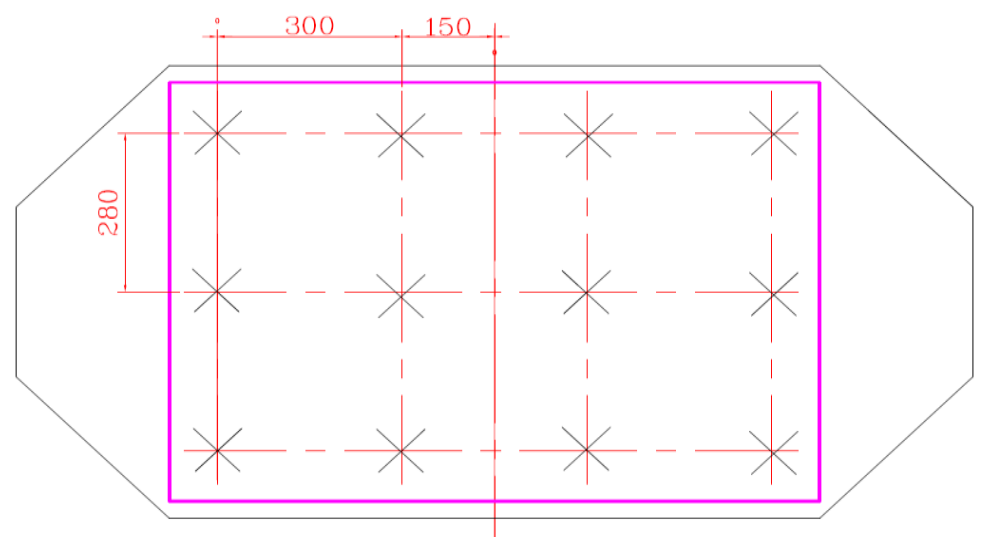

Figure8. SOLE PLATE is divided into 12 points mark schematic 
Table 3 below is a set of raw data of a set of points on a SOLE PLATE measured by a total station:

Table3. Raw data of the collected points on a block of SOLE PLATE

\begin{tabular}{|c|c|c|c|}
\hline \multirow{2}{*}{ Point ID } & \multicolumn{3}{|c|}{ Collected points coordinates } \\
\cline { 2 - 4 } & $x_{i}(\mathrm{~m})$ & $y_{i}(\mathrm{~m})$ & $z_{i}(\mathrm{~m})$ \\
\hline 1 & -99.708 & 29.609 & -8.627 \\
\hline 2 & -99.505 & 29.405 & -8.628 \\
\hline 3 & -99.303 & 29.200 & -8.629 \\
\hline 4 & -99.107 & 28.990 & -8.630 \\
\hline 5 & -99.919 & 29.408 & -8.629 \\
\hline 6 & -99.748 & 29.160 & -8.630 \\
\hline 7 & -99.530 & 28.987 & -8.631 \\
\hline 8 & -99.307 & 28.793 & -8.632 \\
\hline 9 & -100.176 & 29.182 & -8.632 \\
\hline 10 & -99.951 & 28.946 & -8.632 \\
\hline 11 & -99.755 & 28.750 & -8.633 \\
\hline 12 & -99.508 & 28.591 & -8.634 \\
\hline
\end{tabular}

After loading the program in AUTOCAD, use the mouse to directly capture and click on each measurement point to get the vector distance from each measurement point to the optimal plane. After calculation, the vector distance of each point on the SOLE PLATE from the optimal plane is obtained. See Figure 9 below:

\begin{tabular}{|c|c|}
\hline Point ID & Offset distance $H_{i}(\mathrm{~mm})$ \\
\hline 1 & 0.017 \\
\hline 2 & 0.243 \\
\hline 3 & -0.315 \\
\hline 4 & -0.529 \\
\hline 5 & 0.183 \\
\hline 6 & 0.616 \\
\hline 7 & 0.275 \\
\hline 8 & 0.067 \\
\hline 9 & -0.746 \\
\hline 10 & 0.047 \\
\hline 11 & -0.064 \\
\hline 12 & 0.207 \\
\hline
\end{tabular}

Figure9. Vector distance of each point on the measured SOLE PLATE from the optimal plane

From the data in the table, the coplanarity of the SOLE PLATE can be obtained as : $\varepsilon=H_{\text {max }}-H_{\text {min }}=0.616-(-0.746)=1.362 \mathrm{~mm}$.

\subsection{Features of the New Method}

The new method of coplanarity analysis presented in this paper uses the least squares method to fit the optimal plane to determine whether the coplanarity meets the corresponding technical requirements. Through application discovery, the algorithm has the following advantages:

1) Mathematical model construction is clear, easy to understand, simple in form, and easy to be understood by most people.

2) The mathematical model can fully and effectively use all measurement data, which is convenient for computer processing. Use MATLAB or AUTOCAD VBA to write the program, fit the ideal plane, and quickly calculate the vector distance from each measurement point to the optimal plane. The degree of integration is high [10].

3) In the case of large and complex measurement data, the traditional coplanar analysis method is computationally intensive, and the method is a linear problem when using the method for coplanarity analysis. The calculation is simple and the method is not subject to data collection points. And this method is not limited by the number and distribution of data collection points.

4) A wide range of applications. The algorithm can be applied to many aspects of engineering, such as the calculation of the coplanarity of the support plate of the FPSO generator base and the coplanarity analysis of the blind flange of the marine steel structure. 


\section{CONCLUSIONS}

The coplanar analysis methods described in this paper have their own characteristics, and each has its own applicable conditions.

1) When the number of data to be analyzed is small and evenly distributed, the measurement data should be matched to one of the cross-criteria, triangle criterion or linear criterion of the minimum region by linear transformation, so that the traditional coplanarity analysis method is adopted. The three-point method is simpler, but not precise enough, and the result is not unique. The diagonal method is convenient and economical, but the accuracy is not high enough. The minimum area method meets the minimum condition, but the analytical method is difficult to find directly; when the amount of analysis data is small, two parallel planes that meet the minimum condition can be found based on the data and personal experience.

2) When the number of samples to be analyzed is large and the distribution is uneven, the traditional coplanarity analysis method is cumbersome and even impossible to assess. The least squares rule is not subject to this limitation and the surface to be analyzed can be evaluated relatively accurately, and the data processing is a linear problem, and the solution is simple and convenient.

3) Since the new method of the coplanarity analysis is based on the most common simple least squares method, the generalization of the mathematical model is relatively simple. The mathematical model is not only for planes, but also for circles, spheres, cylinders. The same surface is also applicable, corresponding to the flatness, corresponding to roundness, sphericity, and so on.

\section{REFERENCES}

[1] Chen Longde, Zhao Fuling. Mechanical Precision Design and Detection Technology [M]. Beijing: Mechanical Industry Press, 2001.

[2] FeiYetai. Error Theory and Data Processing [J]. Beijing: Mechanical Industry Press, 2004.

[3] Zhang Zhijiang, Yu Yujie, Zhang Shanzhong. A New Algorithm for Minimum Area of Flatness Error-Ordered Discriminant Method [J].Journal of Metrology, 1998, 19 (1): 15 21.

[4] Shi Lixin, ZhuSihong. Evaluation of Flatness Error Minimum Area Method Based on Matlab [J].Modular Machine Tool \& Automatic Processing Technology,2005 (9):58 59.

[5] Liu Shizheng, Zhou Yifang. Pole-point iteration method--a fast and accurate algorithm for finding the minimum conditional flatness error [J].Journal of Applied Science. 1997,15(1):34 40.

[6] Zhang Wei.A Least Squares Analysis of Flatness Error [J].Machinery Manufacturing and Research.2002,7(3):17-19.

[7] Li Minshan, JiChengguang, Lu Honggang, Xiao Wei, Ren Yaoru. Study on Coplanar Measurement Method of Embedded Components [J].Printed Circuit Information.2013,06.

[8] Wang Jinxing, Jiang Xiangqian, Ma Limin, Xu Zhenao, Li Zhu. Calculation of Uncertainty in Flatness Measurement [J]. China Mechanical Engineering. 2005, 19(10), 1701-1703.

[9] Li Shuping. Evaluation of Flatness Error Least Squares Based on EXCEL [J].Journal of Guangxi University of Technology, 2006,06.

[10] Chen Gongtong. A method for measuring the coplanarity of terminals [P].2015.

[11] ISO/TS 17450-1. Geometrical Product Specification (GPS)-General Concept-Part 1: Model for Geometric Specification and Verification.2002.

[12] ISO/TS 17450-1. Geometrical Product Specification (GPS)-General Concept-Part 2: Basic Tenets, Specifications, Operators and Uncertainties.2002.

[13] ISO 14253-1, Geometrical Product Specification (GPS)- inspection by Measurement of Work pieces and Measuring Equipment -Part 1: Decision Rulers for Proving Conformance or Non-conformance with Specifications.1998.

Citation: Meng Linghe, et.al (2019). "Discussion on Analysis Method of Coplanarity of Marine Steel Structure", International Journal of Petroleum and Petrochemical Engineering (IJPPE), 5(1), pp.16-23, DOI: http://dx.doi.org/10.20431/2454-7980.0501003

Copyright: (0) 2019 Authors. This is an open-access article distributed under the terms of the Creative Commons Attribution License, which permits unrestricted use, distribution, and reproduction in any medium, provided the original author and source are credited 